\title{
Microfiltration de lactosérum doux sur membranes d'alumine : influence des conditions hydrodynamiques sur le colmatage
}

\author{
M Pouliot ${ }^{1}$, Y Pouliot ${ }^{1}$, M Britten ${ }^{2}$, N Rodrigue 2 \\ 1 Centre de recherche STELA, département de sciences et technologie des aliments, \\ université Laval, Québec, Canada G1K 7P4; \\ 2 Centre de recherche et de développement sur les aliments, \\ Agriculture Canada, Saint-Hyacinthe, Canada J2S 8E3
}

(Reçu le 11 juin 1993 ; accepté le 19 janvier 1995)

\begin{abstract}
Résumé - ll est possible de caractériser le colmatage d'un microfiltre par l'évaluation de la résistance hydraulique décomposée en une série de résistances partielles en cours de microfiltration. Différentes conditions hydrodynamiques ( 4 pressions transmembranaires et 3 vitesses tangentielles) ont été étudiées lors de la microfiltration de lactosérum pour vérifier leur effet sur le colmatage et la rétention protéique d'un système à 4 membranes de microfiltration en alumine (Ceraflo, $0,22 \mu \mathrm{m}$ de diamètre de pore) montées en série. Le colmatage total a été significativement affecté par la pression transmembranaire, le facteur de concentration volumique et la vitesse tangentielle. La pression transmembranaire a constitué le facteur le plus important. La rétention $(\sigma)$ de la sérum albumine bovine était totale, la $\beta$ lactoglobuline était retenue à $>0,55$ et l' $\alpha$-lactalbumine à 0,40 dès le début de la filtration. La rétention était surtout affectée par la pression transmembranaire et la concentration des solutés et très peu par la vitesse tangentielle. La géométrie du filtre et les résultats expérimentaux suggèrent que le colmatage se situe surtout à l'intérieur de la matrice microporeuse à cause de la forte influence de la pression transmembranaire et de la faible influence de la vitesse tangentielle. Le colmatage serait dû à la déposition d'agrégats protéiques sous l'effet dénaturant des forces de cisaillement.
\end{abstract}

microfiltration / membrane céramique / résistance hydraulique / rétention protéique / colmatage / lactosérum / conditions hydrodynamiques

Summary - Microfiltration of sweet whey on alumina membranes: influence of the hydrodynamic conditions on the fouling. It is possible to characterize the fouling of a microfilter by evaluating the hydraulic resistance, as made of a series of partial resistances during microfiltration. Different hydrodynamic conditions ( 4 transmembrane pressures and 3 tangential flows) have been studied during whey microfiltration on a system made of 4 alumina membranes (Ceraflo, $0.22 \mu \mathrm{m}$ pore diameter) connected in series. The hydraulic resistance of the membranes during concentration was considered to represent the fouling produced by adsorption, pore blocking and concentration polarization. The effects 
of transmembrane pressure and tangential velocity were analysed in terms of how they affect the fouling. The total fouling was significantly affected by the transmembrane pressure, the volume concentration factor and the tangential flow. The transmembrane pressure represented the most important factor. Bovine serum albumin retention was total, $\beta$-lactoglobulin was retained at $>0.55$ and $\alpha$-lactalbumin at 0.40 at the beginning of the filtration. The results were examined in terms of how the fouling could affect the retention of the 3 main whey proteins. Retention was mainly affected by the transmembrane pressure, by the solutes concentration and very little by tangential flow. The geometry of the filter and the experimental results suggest that the fouling is mainly present inside the macroporous matrix. The strong influence of the transmembrane pressure together with the weaker influence of the tangential flow on the parameters under study suggest that fouling is mainly present in depth of the membrane. The fouling would be due to the deposition of protein aggregates produced by the denaturing effect of the shear forces.

microfiltration / ceramic membrane / fouling / whey / hydraulic resistance / protein retention / hydrodynamic conditions

\section{INTRODUCTION}

La microfiltration tangentielle est utilisée en technologie alimentaire pour la clarification de jus et pour la stérilisation à froid de différentes boissons (Van Der Horst et Hanemaaijer, 1990). Dans le secteur laitier, la microfiltration peut être utilisée pour débarrasser les laits de fromagerie des bactéries et spores nuisibles (Trouvé et al, 1991), pour la clarification et la délipidation du lactosérum (Merin et Daufin, 1990) et pour l'écrémage et la standardisation des laits de consommation (Olesen et Jensen, 1989). Cependant, l'introduction à grande échelle des filtrations tangentielles est limitée par les problèmes de colmatage des membranes (Van Der Horst et Hanemaaijer, 1990). Ceux-ci font diminuer les flux de perméation, modifier la rétention, et augmenter les coûts de nettoyage (Cheryan, 1986; Daufin et al, 1992).

II est reconnu que le colmatage d'une membrane de microfiltration résulte de 3 phénomènes qui se produisent plus ou moins simultanément: 1) adsorption de solutés au niveau du matériau membranaire ; 2) formation de concentration de polarisation et de gâteau de filtration en surface du filtre, et 3 ) blocage des pores en surface et en profondeur (Van Der Horst et Hanemaaijer, 1990).
De nombreuses études de colmatage ont été réalisées avec des solutions simples de protéines purifiées resuspendues dans des tampons (Opong et Zydney, 1991 ; Bowen et Gan, 1991a, 1991b et 1992) et des fluides alimentaires (Mietton-Peuchot et al, 1984 ; Attia et al, 1991a, b ; Belleville et al, 1992) afin d'évaluer certaines conditions physicochimiques et hydrodynamiques favorisant le colmatage des membranes.

La sélectivité des membranes de microfiltration a souvent été examinée en présence de solutions simples de protéines purifiées (Bowen et Hughes, 1990 ; Bowen et Gan, 1991 a,b, 1992 et 1993 ; Kelly et al, 1993). Quelques phénomènes ont ainsi pu être mis en évidence en microfiltration et en ultrafiltration tels que l'effet du cisaillement, de la température et de la concentration sur la susceptibilité de certaines protéines à s'adsorber ou à former des agrégats (Meireles et al, 1991 ; Bowen et Gan, 1991a, b et 1992; Kim et al, 1993). On trouve peu d'études tentant de mettre en évidence ces mêmes phénomènes dans des systèmes complexes tels que les fluides laitiers (Gésan, 1993).

Les membranes minérales se présentent souvent comme une couche de particules minérales de fine granulométrie, définissant le diamètre de pores de la membrane, déposée sur un support plus grossier (Vétier et al, 
1986 ; Chan et Brownstein, 1991 ; Charpin et al, 1991). Selon le fabricant de la membrane, cette couche a une épaisseur d'environ 20 à $25 \mu \mathrm{m}$, ce qui pour une membrane de 0,22 $\mu \mathrm{m}$ de diamètre de pores représente un rapport longueur de pore sur diamètre de pore d'environ 100. Étant donné la tortuosité des pores, le rapport longueur/diamètre est probablement beaucoup plus élevé, ce qui, dans le cas de petits solutés, représente un filtre en profondeur.

Une description exacte de la filtration devrait idéalement tenir compte du caractère géométrique et physique du filtre (taille et forme des pores, propriétés de surface, etc), des propriétés du fluide (viscosité, teneur en solutés, géométrie des solutés, etc) et de l'hydrodynamique de l'écoulement (pression transmembranaire, vitesse tangentielle turbulence etc) (Azbel et Cheremisinoff, 1983). Plusieurs équations ont été développées afin de modéliser le flux de perméation de différents procédés en cours de filtration (Cheryan, 1986 ; Taddei et al, 1989 ; Lahoussine-Turcaud et al, 1990 ; Nilsson, 1990 ; Warren et al, 1992). En pratique, la complexité de tels modèles augmente rapidement et une évaluation exacte de plusieurs de ces paramètres est difficile voire impossible. II existe cependant des modèles plus accessibles, tel le modèle de résistance en série (Taddei et al, 1988 en ultrafiltration ; Gésan, 1993 en microfiltration) qui décompose la résistance hydraulique du système en une série de résistances partielles :

$$
\mathrm{J}=\frac{\mathrm{Pt}}{\mathrm{Rh} \cdot \mu}
$$

où $\mathrm{J}$ : flux de perméation; $\mathrm{Pt}$ : pression transmembranaire ; $\mathrm{Rh}$; résistance hydraulique du filtre ; $\mu$ : viscosité dynamique du filtrat ; et où

$$
\mathrm{Pt}=\frac{(\mathrm{P} \text { entrée }+\mathrm{P} \text { sortie })}{2}-\mathrm{P} \text { perméat }
$$

D'autre part, l'équation de Poiseuille décrit l'écoulement à travers un capillaire droit et lisse d'un fluide visqueux et incompressible sous régime laminaire selon:

$$
J=\frac{e d p^{2}}{32} \frac{P t}{\Delta x \mu}
$$

où $e:$ porosité du filtre $; \mathrm{dp}$ : diamètre de pores ; $\Delta \mathrm{x}$ : longueur du pore.

En combinant (1) et (2), on peut décrire la résistance hydraulique d'un système de filtration comme :

$$
R h=\frac{32 \Delta x}{e d p^{2}}
$$

II est ainsi possible d'entrevoir quels paramètres déterminent la résistance hydraulique d'une membrane. L'adsorption de solutés diminue le diamètre de pores, la concentration de polarisation et le gâteau de filtration augmentent l'épaisseur du filtre, et le blocage des pores diminue la porosité. Ces phénomènes conduisent à une augmentation de la résistance hydraulique, d'où une perte de flux. L'adsorption de solutés est fonction des conditions physico-chimiques des solutés du fluide, la concentration de polarisation et le gâteau de filtration sont fonction de la vitesse tangentielle d'écoulement à la surface du filtre (et donc de la turbulence) et le blocage des pores est fonction de la convection des solutés vers la surface de la membrane. Nos travaux antérieurs portant sur la microfiltration du lactosérum ont mis en évidence un phénomène de colmatage interne des membranes d'alumine par les protéines du lactosérum. Cela supporte l'hypothèse de Bowen et Gan (1991b) qu'en microfiltration le colmatage est beaucoup plus susceptible d'être dû à l'adsorption de protéines en profondeur du filtre qu'en ultrafiltration puisque dans cette première une fraction des protéines peut traverser la membrane. 
Le colmatage en profondeur pourrait être responsable de la haute rétention protéique observée par Piot et al (1984) lors de la microfiltration de lactosérum sur différentes membranes de microfiltration. D'autres études en microfiltration (Attia et al, 1991a, b) rapportent des rétentions de 0,95 pour la sérum albumine bovine (SAB), 0,70 pour la $\beta$-lactoglobuline et de 0,35 pour l' $\alpha$-lactalbumine lors de la microfiltration de lait sur membrane minérale de $0,2 \mu \mathrm{m}$. De même, Pierre et al (1992) ont observé une rétention de l'azote non caséique (NCN) de l'ordre de 0,40 lors de la préparation de phosphocaséinate natif à faible pression transmembranaire.

Concernant la composition (Marshall et Harper, 1988), la fraction protéique du lactosérum représente 5,5 à $6,5 \mathrm{~g} \mathrm{|}^{-1}$ et se compose principalement de $\beta$-lactoglobuline $(55-65 \%)$, d' $\alpha$-lactalbumine (15-25\%), d'immunoglobulines $(10-15 \%)$, de protéoses-peptones (10-20\%) et de sérum albumine $(5-6 \%)$. Cependant, en termes physico-chimiques, ces fractions se distinguent par leur masse moléculaire et leur volume hydrodynamique, et par leur point isoélectrique.

La SAB possède une masse de 67000 , avec un point isoélectrique d'environ 4,8 et des dimensions de $11,6 \times 2,7 \times 2,7 \mathrm{~nm}$ (Bowen et Gan, 1991a). La $\beta$-lactoglobuline se présente sous forme dimérique entre les $\mathrm{pH}$ de 5 à 8 , de masse de 36000 , avec un point isoélectrique de 5,18 et un rayon de Stokes de 2,68 nm (Hambling et al, 1992). L' $\alpha$-lactalbumine possède une masse de 14200 , avec un point isoélectrique de 4,8 et les dimensions de $2,3 \times 2,6 \times 4,0 \mathrm{~nm}$ (Brew et Grobler, 1992). Enfin, les immunoglobulines et protéosespeptones constituent un groupe de composés protéiques moins bien caractérisés. On sait cependant (Marshall et Harper, 1988) que les immunoglobulines possèdent une masse moléculaire pouvant varier entre 80000 et 900000 et un point iso- électrique entre 5,5 et 8,3 . Quant aux protéoses-peptones, leurs masses moléculaires varient entre 4000 et 80000 et leurs points isoélectriques entre 5,1 et 6,0 .

Le lactosérum présente donc une composition hétérogène du point de vue de la nature et des propriétés physicochimiques de ses protéines. L'état actuel des connaissances sur la microfiltration du lactosérum suggère une importante contribution des conditions hydrodynamiques (pression transmembranaire et vitesse tangentielle) à la rétention spécifique de certaines fractions protéiques.

Le but du présent travail était de vérifier l'impact des conditions hydrodynamiques (pression transmembranaire et vitesse tangentielle) sur le colmatage des membranes d'alumine lors de la microfiltration de lactosérum de fromagerie par l'évaluation de la résistance hydraulique des membranes ainsi que sur la rétention des protéines.

\section{MATÉRIEL ET MÉTHODES}

\section{Banc d'essai}

Le banc d'essai était constitué de 4 membranes tubulaires d'alumine (Ceraflo, Millipore) de $0,22 \mu \mathrm{m}$ de diamètre de pore. Les 4 membranes étaient connectées en série à une pompe à déplacement positif à vitesse variable (Cole Palmer Instruments $\mathrm{Co}$, Chicago, États-Unis); leur diamètre hydraulique était de $3,0 \mathrm{~mm}$ pour une surface filtrante de $110^{-3} \pm 410^{-5} \mathrm{~m}^{2}$ par membrane. Des manomètres étaient installés à l'entrée et à la sortie de chaque membrane, ainsi qu'une vanne à la sortie de chaque membrane. Cela permettait d'obtenir, dans chacune des 4 membranes, 4 pressions transmembranaires moyennes $(179,138,97$ et $55 \mathrm{kPa} \pm 4 \mathrm{kPa})$ pour chacune des 3 vitesses tangentielles $(2,0,3,0$ et $4,0 \mathrm{~m} \mathrm{~s}^{-1} \pm 6 \%$ ) utilisées. Un serpentin d'acier inoxydable plongé dans un bain thermostaté permettait de faire les microfiltrations à $50 \pm 1^{\circ} \mathrm{C}$ (fig 1). Le volume mort de l'appareil s'élevait à $300 \mathrm{ml}$. 


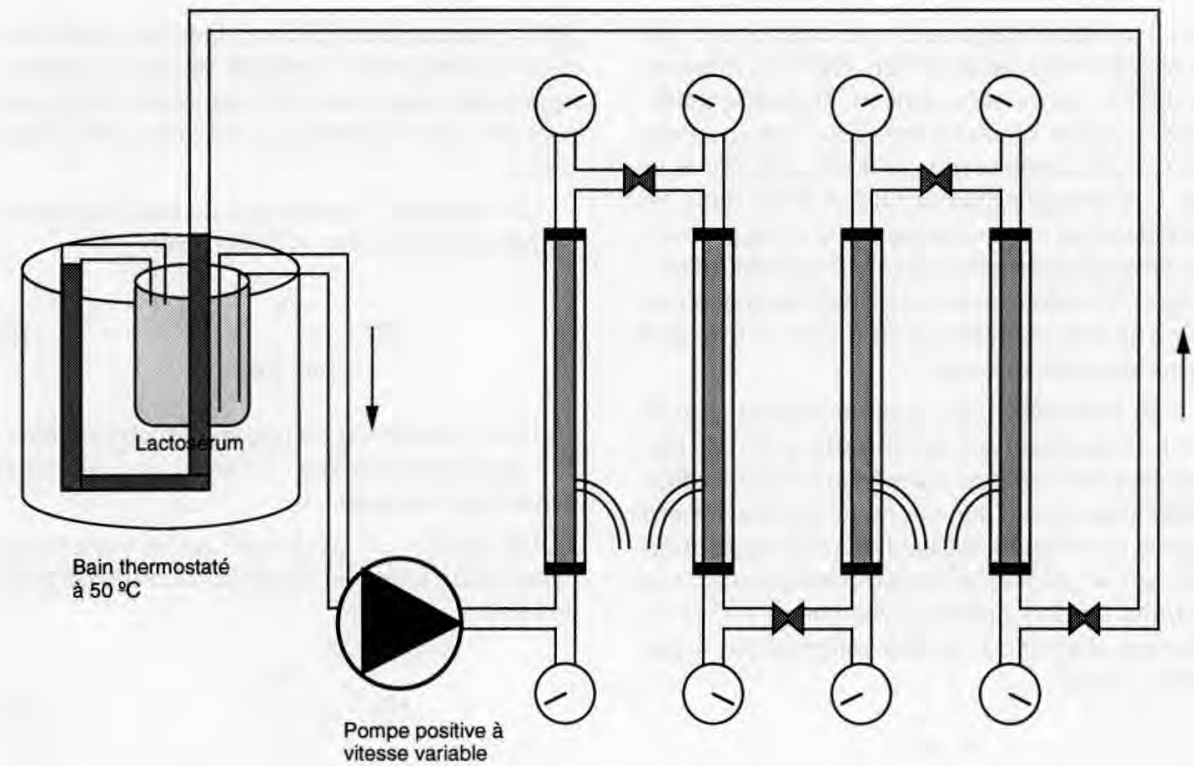

Fig 1. Banc d'essai de microfiltration à vitesse tangentielle constante.

Constant tangential flow microfiltration work bench.

Plusieurs membranes ont été assemblées et mises sur banc d'essai après avoir subi la séquence de lavage : 15 min sous $\mathrm{HNO}_{3} 2 \%$, 10 min de rinçage, 15 min sous $\mathrm{NaOH} 2 \%, 10$ min de rinçage, $15 \mathrm{~min}$ sous $\mathrm{NaOCl}(200 \mathrm{ppm}$ de chlore résiduel total) et $15 \mathrm{~min}$ de rinçage avant la mesure du flux de perméation à l'eau. Toutes les solutions étaient préparées dans de l'eau distillée déionisée avec des produits de grade réactifs et étaient recirculées à $50^{\circ} \mathrm{C}$. Quatre membranes ont été sélectionnées et montraient un flux moyen de $1550 \pm 110 \mathrm{I} \mathrm{h}^{-1} \mathrm{~m}^{-2}$ (ie $R m$ de $4,810^{10} \pm 510^{9} \mathrm{~m}^{-1}$ à $5,210^{10} \pm 510^{9}$ $\mathrm{m}^{-1}$ ) sous les conditions de $186 \mathrm{kPa}$ de pression transmembranaire, $4,0 \mathrm{~m} \mathrm{~s}^{-1}$ de vitesse tangentielle à $26^{\circ} \mathrm{C}$ après au moins $15 \mathrm{~min}$ de rinçage à l'eau distillée déionisée.

Les vitesses tangentielles moyennes ont été estimées par mesure du débit volumétrique de la pompe rapporté sur la section membranaire. Les vitesses de 2,0,3,0 et $4,0 \mathrm{~m} \mathrm{~s}^{-1}$ équivalaient à des nombres de Reynolds respectifs de 10500 , 15700 et 21000 pour de l'eau distillée déionisée à $50^{\circ} \mathrm{C}$. Les flux de perméation ont été calculés à partir des débits volumétriques de perméation rapportés sur la surface filtrante.

\section{Essais}

Le lactosérum était préparé à partir de poudre de lactosérum de grand mélange (Agrinove, SteClaire, Canada) réhydratée à $7,0 \%(\mathrm{p} / \mathrm{v})$ dans de l'eau distillée déionisée avec addition de $0,02 \%$ de $\mathrm{NaN}_{3}$. Les solutions contenaient en moyenne $1,06 \pm 0,03 \%$ de protéines totales (mesurées par spectroscopie infra-rouge), $0,32 \pm 0,01 \%$ d'azote non protéique (NPN) soluble au TCA $12 \%$ (méthode Kjeldahl, facteur de 6,38) avec 0,076 \pm $0,004 \%$ de matières grasses résiduelles (méthode Mojonnier). Les poudres étaient réhydratées la veille de la microfiltration, gardées sous agitation à $7^{\circ} \mathrm{C}$, puis préchauffées à $50^{\circ} \mathrm{C}$ avant la microfiltration. Les solutions avaient toutes un $\mathrm{pH}$ de $5,65 \pm 0,01$ en début de filtration et montraient une légère fraction insoluble.

Avant et après chaque essai de microfiltration, le système était nettoyé selon la séquence: $15 \mathrm{~min}$ de $\mathrm{NaOH} 2 \%$ en recirculation totale, $5 \mathrm{~min}$ de rinçage sans recirculation, 15 min de $\mathrm{HNO}_{3}$ et/ou $\mathrm{H}_{3} \mathrm{PO}_{4} 2 \%$ en recirculation, $5 \mathrm{~min}$ de rinçage sans recirculation, $15 \mathrm{~min}$ de $\mathrm{NaOCl}$ (200 ppm de chlore résiduel total) en recirculation 
puis $5 \mathrm{~min}$ de rinçage sans recirculation et $5 \mathrm{~min}$ de rinçage avec recirculation avant les mesures de flux de perméation à l'eau. Toutes les solutions utilisées étaient préparées avec de l'eau distillée déionisée et des produits de grade réactifs. Les lavages étaient faits à $50^{\circ} \mathrm{C}$ dans les conditions de vitesse tangentielle et de pression transmembranaire de l'essai de microfiltration à réaliser. La séquence était répétée jusqu'à obtention d'un flux de perméation à l'eau $\pm 8 \%$ de la valeur du premier essai.

Les microfiltrations étaient réalisées avec 1,2 I de lactosérum préchauffé à $50^{\circ} \mathrm{C}$. Des mesures de débit de perméation étaient effectuées toutes les 5 min sur chacune des 4 membranes permettant le calcul des flux de perméation aux 4 pressions transmembranaires. Les facteurs de concentration volumique (FCV) ont été calculés par cumul des volumes des 4 perméats, selon :

\section{$\mathrm{Ri}$}

$$
F C V=\overline{R i-(P \# 1+P \# 2+P \# 3+P \# 4)}
$$

où $\mathrm{Ri}$ : volume de rétentat initial ; $\mathrm{P}$ : volume de perméat au temps $\mathrm{t}$; \# : position de la membrane.

La concentration était poussée jusqu'à un facteur volumique de $3 \mathrm{X}$ qui correspondait à 120 à $160 \mathrm{~min}$ de filtration selon la vitesse tangentielle.

Des échantillons de perméats et de rétentats étaient recueillis pour toutes les valeurs de FCV entières afin d'en mesurer la viscosité dynamique des perméats à $50^{\circ} \mathrm{C}$ sur un viscosimètre Carrimed Controlled Stress Rheometer (Carrimed Ltd, Dorking, Angleterre), les protéines totales par spectroscopie infrarouge (Milko Scan 133B spectromètre à infrarouge moyen, Foss Electric, Danemark) et le profil protéique par chromatographie HPLC de tamisage moléculaire. Pour l'analyse HPLC, l'échantillon était dilué à $0,05 \%$ de protéines dans le tampon d'élution et 20 $\mu$ étaient injectés sur une colonne Bio-Sil ${ }^{\circledast}$ SEC 125 (7.8 mm x $30 \mathrm{~cm}$; Bio-Rad,Richmonds, CA, États-Unis). Le système de chromatographie était composé d'un module pour solvants (modèle 126 ), d'un module de détection UV (modèle 166), et d'un logiciel de traitement de données (System Gold ${ }^{T M}$; Beckman Instruments, San Ramon, $\mathrm{CA}$, États-Unis). La colonne était éluée avec $\mathrm{KH}_{2} \mathrm{PO}_{4}\left(0,015 \mathrm{~mol} \mathrm{I}^{-1}, \mathrm{pH} 6,5\right)$ à un débit de $0,3 \mathrm{ml} \mathrm{min}^{-1}$. Les protéines étaient détectées par absorption en UV à $212 \mathrm{~nm}$. La concentration de
SAB, $\beta$-lactoglobuline et $\alpha$-lactalbumine était déterminée par les surfaces de pics. L'étalonnage a été réalisé avec des standards protéiques purs de Sigma Chemical Co (St-Louis, MO, EtatsUnis).

La résistance hydraulique du matériau membranaire propre a été évaluée selon :

$$
\mathrm{Rm}=\frac{\mathrm{Pt}}{\text { Je. } \mu \mathrm{e}}
$$

où $\mathrm{Rm}$ : résistance du matériau membranaire ; Je : flux de perméation à l'eau ; $\mu$ e : viscosité dynamique de l'eau.

De même, on peut évaluer la résistance hydraulique totale du système en cours de filtration par :

$$
\mathrm{Rh}=\frac{\mathrm{Pt}}{\mathrm{J} \cdot \mu}
$$

où $\mathrm{J}$ : flux de perméation en filtration ; $\mu$ : viscosité dynamique du perméat.

avec

$$
R h=R m+R c
$$

où Rc : résistance due au colmatage.

On peut alors exprimer la résistance due au colmatage par :

$$
\mathrm{RC}=\frac{\mathrm{Pt}}{\mathrm{J} \cdot \mu}-\frac{\mathrm{Pt}}{\mathrm{Je} \cdot \mu \mathrm{e}}
$$

La rétention des protéines était calculée selon :

$$
\sigma=1-\mathrm{Cp} / \mathrm{Cr} ;
$$

oũ $\mathrm{Cp}=$ concentration en soluté du perméat ; $\mathrm{Cr}=$ concentration en soluté du rétentat.

Pour chaque vitesse tangentielle, 3 filtrations ont été conduites selon un plan expérimental en tiroirs subdivisés pour un total de 9 filtrations, avec en parcelles principales la vitesse, en sousparcelles le facteur de concentration et en soussous-parcelles la pression transmembranaire. Des analyses de variance (Anova) ont été conduites sur les valeurs de résistances hydrauliques et de rétention protéique selon un test de Fisher. Des analyses de contrastes ont été menées pour permettre la modélisation, ainsi qu'un test d'ajustement pour vérifier les modèles produits (Steel et Torrie, 1980). 


\section{RÉSULTATS}

\section{Résistance du matériau membranaire propre}

Pour les 4 membranes en série ayant subi la même séquence de lavage, les flux de perméation à l'eau variaient entre $950 \mathrm{I}$ $\mathrm{h}^{-1} \mathrm{~m}^{-2}$ à $55 \mathrm{kPa}$ et $1600 \mathrm{I} \mathrm{h}^{-1} \mathrm{~m}^{-2}$ à $179 \mathrm{kPa}$ à $50^{\circ} \mathrm{C}$, soit une augmentation du flux de 1,7 fois pour une augmentation de pression de 3 fois. L'analyse de variance des valeurs de résistance membranaire $(R \mathrm{~m})$ calculées à partir des flux à l'eau des membranes propres indique que seule la pression transmembranaire a un effet significatif $(P<0,05)$ sur la résistance membranaire, la vitesse tangentielle n'ayant aucun effet significatif sur $\mathrm{Rm}$. Ces résultats ne respectent pas la loi de Darcy, qui prédit que Rm est constant en fonction de la pression. Les valeurs de $\mathrm{Rm}$ ont quand même été conservées, puisqu'elles représentent une mesure instantanée de l'état de la membrane au début de l'expérience.

L'analyse des contrastes de $\mathrm{Rm}$ indique que la pression transmembranaire a un effet

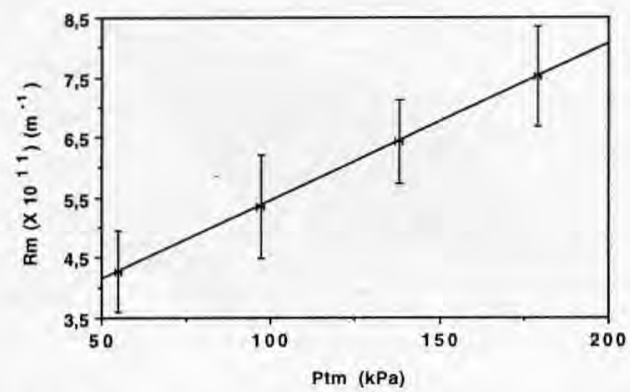

Fig 2. Évolution de la résistance hydraulique membranaire $(\mathrm{Rm})$ avec la pression transmembranaire $(\mathrm{Pt})$ de membranes d'alumine (Ceraflo, $0,22 \mu \mathrm{m}$ ) propres, calculée à partir des flux de perméation à l'eau pure (Jw).

Membrane hydraulic resistance $(R m)$ evolution of clean alumina membrane (Ceraflo, $0.22 \mu \mathrm{m}$ ) with the transmembrane pressure $(P t)$, determined by pure water flux measurement (Jw). fortement linéaire et légèrement cubique sur l'augmentation de Rm. Nous n'avons conservé que la composante linéaire pour la modélisation de l'évolution de $\mathrm{Rm}\left(\mathrm{m}^{-1}\right)$ avec la pression (fig 2). Le modèle produit s'ajuste bien aux données expérimentales au seuil de $P>0,05$.

\section{Flux de perméation en cours de microfiltration}

Les courbes de flux de perméation en fonction du FCV sont présentées sur la figure 3 (A à C). Ainsi, des valeurs de flux similaires sont obtenues à 179,138 et $97 \mathrm{kPa}$. L'effet de la vitesse tangentielle se traduit par une augmentation du flux moyen de $85 \mathrm{I} \mathrm{h}^{-1} \mathrm{~m}^{-2}$ à $2 \mathrm{~m} \mathrm{~s}^{-1}$ (fig $3 A$ ) à $95 \mathrm{I} \mathrm{h}^{-1} \mathrm{~m}^{-2}$ à $3 \mathrm{~m} \mathrm{~s}^{-1}$ (fig 3B) jusqu'à $105 \mathrm{I} \mathrm{h}^{-1} \mathrm{~m}^{-2}$ à $4 \mathrm{~m} \mathrm{~s}^{-1}$ (fig $3 \mathrm{C}$ ). La diminution des flux de perméation pendant l'augmentation du FCV de 1 à 3 est limitée à environ $5 \mathrm{I} \mathrm{h}^{-1} \mathrm{~m}^{-2}$ dans toutes les conditions de pression et de vitesse tangentielle étudiées.

L'augmentation de la pression transmembranaire d'un facteur supérieur à 3 ne permet qu'une augmentation de flux d'environ 1,25 fois, tandis qu'une augmentation de 2 fois de la vitesse tangentielle provoque une augmentation de 1,2 à 1,4 fois du flux de perméation.

\section{Résistance hydraulique en cours de microfiltration}

La résistance hydraulique totale augmente rapidement avec la pression dans un rapport près de 1:1, alors que l'augmentation de FCV n'augmente Rh que de 1,5 fois durant une concentration de $3 X$. La vitesse tangentielle a aussi un effet limité sur Rh, montrant une légère baisse malgré une vitesse 2 fois plus élevée.

L'analyse de variance des résistances hydrauliques totales calculées à partir des 


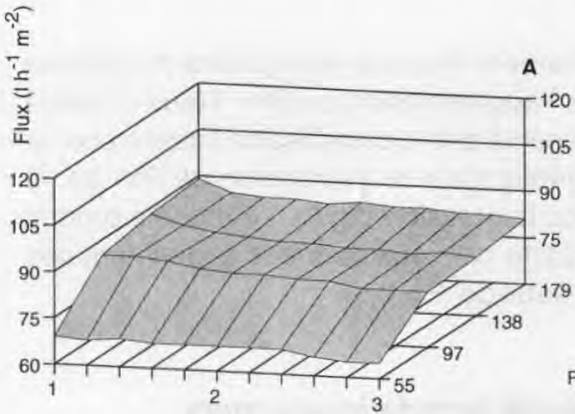

FCV
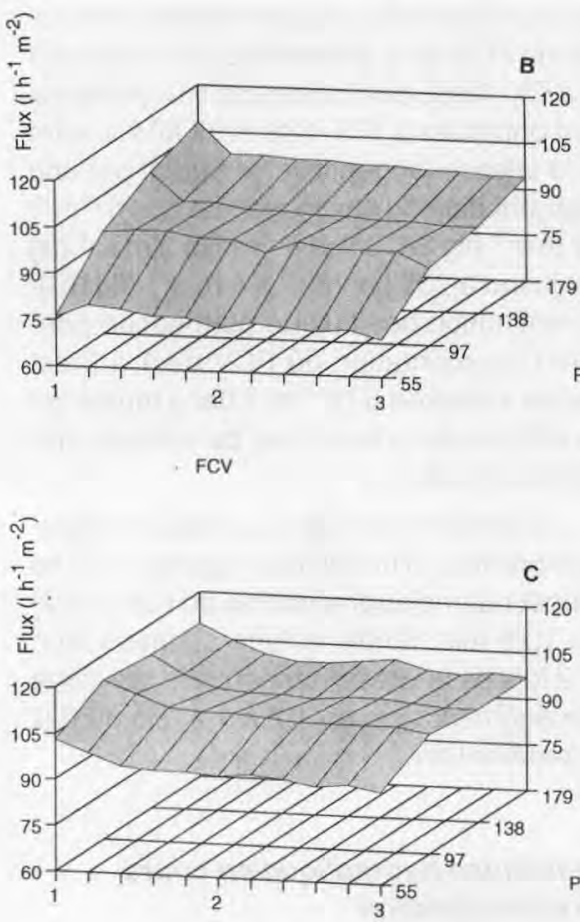

FCV

Fig 3. Évolution des flux de perméation (J) en cours de microfiltration de lactosérum en fonction du facteur de concentration volumique (FCV) et de la pression transmembranaire $(\mathrm{Pt})$ aux vitesses tangentielles de: $\mathrm{A}, 2 \mathrm{~m} \mathrm{~s}^{-1}, \mathrm{~B}, 3 \mathrm{~m} \mathrm{~s}^{-1}$ et C, $4 \mathrm{~m} \mathrm{~s}^{-1}$.

Permeation flux $(\mathrm{J})$ evolution during microfiltration of whey versus the volume concentration factor (FCV) and the transmembrane pressure (Pt) at tangential flows of: $A, 2 \mathrm{~m} \mathrm{~s}^{-1}, B, 3 \mathrm{~ms}^{-1}$ et $C$, $4 \mathrm{~m} \mathrm{~s}^{-1}$. flux de perméation indique qu'il n'y a que des effets linéaires $(P<0,05)$ des répétitions, de la vitesse tangentielle (V), du facteur de concentration (FCV) et de la pression transmembranaire (Pt) sur l'évolution de la résistance hydraulique totale ( $\mathrm{Rh}$ ), la pression étant la variable la plus statistiquement significative. II n'y a aucune interaction double significative entre les différentes variables.

L'analyse des contrastes de $\mathrm{Rh}$ indique que seuls les effets linéaires de $\mathrm{V}$, de FCV et de $\mathrm{Pt}$ sont significatifs $(P<0,05)$. Nous avons donc pu modéliser l'évolution de Rh $\left(\mathrm{m}^{-1}\right)$ par:

$$
\begin{aligned}
\mathrm{Rh}=6,07 & \times 10^{11}-1,91 \times 10^{11 *} \mathrm{~V}+9,58 \\
& \times 10^{10 *} \mathrm{FCV}+1,48 \times 10^{9 *} \mathrm{Pt}
\end{aligned}
$$

qui s'ajuste bien, au seuil de $P>0,05$, aux données expérimentales (fig 4).

\section{Résistance hydraulique due au colmatage}

La résistance hydraulique due au colmatage $(R c)$ est obtenue par la différence entre

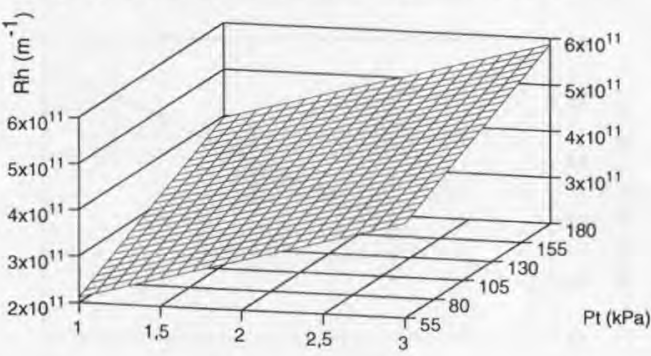

FCV

Fig 4. Évolution de la résistance hydraulique totale (Rh) en cours de microfiltration de lactosérum en fonction du facteur de concentration volumique (FCV) et de la pression transmembranaire $(\mathrm{Pt})$ à la vitesse tangentielle de $3 \mathrm{~m} \mathrm{~s}^{-1}$. Total hydraulic resistance evolution during microfiltration of whey versus the volume concentration factor (FCV) and the transmembrane pressure $(P t)$ at a tangential flow of $3 \mathrm{~m} \mathrm{~s}^{-1}$. 
la résistance hydraulique totale $(\mathrm{Rh})$ et la résistance du matériau membranaire $(\mathrm{Rm})$ obtenue du modèle. Comme Rh, Rc augmente peu avec le FCV et $V$ a un léger effet quadratique. À des fins d'analyse de variance, les valeurs de $R c$ ont subi une transformation logarithmique afin d'homogénéiser la variance. Les variables et interactions significatives $(P<0,05)$ sont: $\mathrm{V}, \mathrm{V}$, $\mathrm{FCV}, \mathrm{Pt}, \mathrm{Pt}^{2}, \mathrm{VxPt}$ et $\mathrm{V}^{2} \mathrm{xPt}, \mathrm{Pt}$ étant la variable la plus significative. L'analyse de variance a permis de construire des surfaces de régressions décrivant bien les données expérimentales au seuil de $P>0,05$ (fig 5).

\section{Rétention des protéines}

La poudre de lactosérum réhydratée contient en plus des 3 protéines principales (SAB, $\beta$-lactoglobuline et $\alpha$-lactalbumine) plusieurs fractions de faible masse moléculaire qui seraient associées à la présence de peptides provenant de l'hydrolyse par la présure, la plasmine et les enzymes microbiennes des caséines et

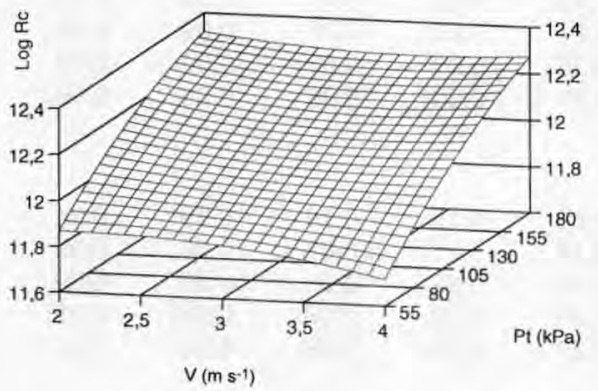

Fig 5. Évolution de la résistance hydraulique due au colmatage ( $\log R c)$ en cours de microfiltration de lactosérum en fonction de la vitesse tangentielle (V) et de la pression transmembranaire (Pt) à un facteur de concentration volumique (FCV) de 2. Evolution of the hydraulic resistance ( $\log R c)$ caused by fouling during microfiltration of whey versus the tangential velocity $(V)$ and the transmembrane pressure (Pt) at a volume concentration factor (FCV) of 2. des protéines sériques (fig 6A). La quantité de matériel protéique total injecté sur la colonne HPLC a été la même pour le rétentat et le perméat, si bien que les peptides se retrouvaient en proportion plus élevée dans les perméats indiquant une faible rétention (fig $6 \mathrm{~B}$ ). Les protéines volumineuses ( $\mathrm{SAB}$ ) ont été complètement retenues par la membrane et sont absentes du perméat.
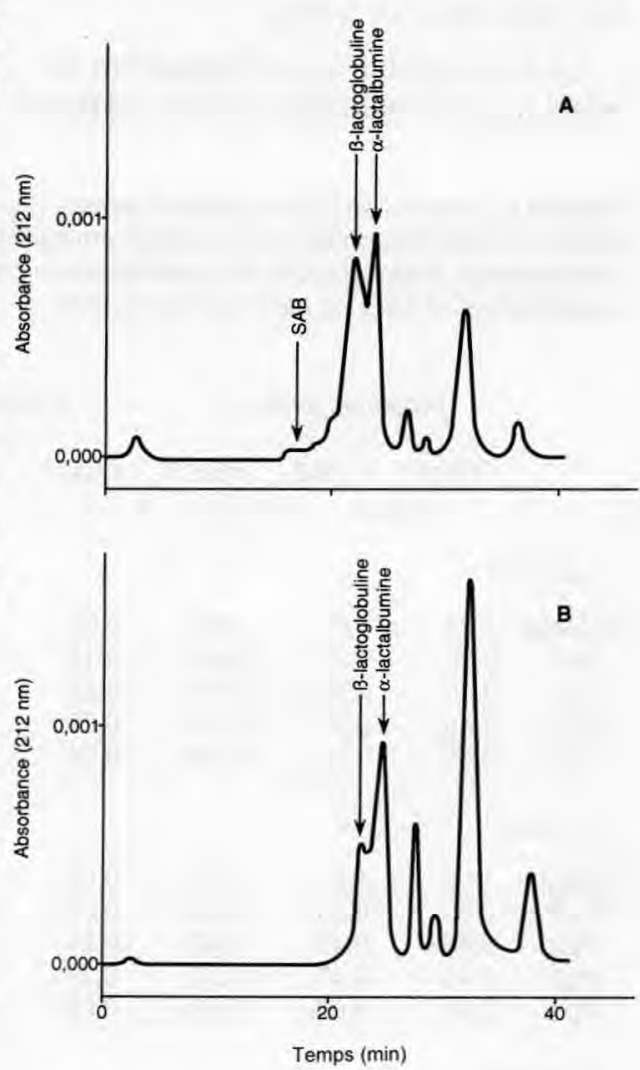

Fig 6. Profil de chromatographie HPLC sur colonne BIO-Sil SEC 25. Élution avec $\mathrm{KH}_{2} \mathrm{PO}_{4}$ $0,015 \mathrm{~mol} \mathrm{I}^{-1}, \mathrm{pH} 6,5$ et détection à $212 \mathrm{~nm}$. A) lactosérum réhydraté, $B$ ) perméat de microfiltration.

HPLC chromatographic profile on BIO-Sil SEC 25 column. Elution with $\mathrm{KH}_{2} \mathrm{PO}_{4} 0.015 \mathrm{~mol}^{-1}$, $\mathrm{pH} 6.5$ and detection at $212 \mathrm{~nm}$. A) rehydrated whey, B) microfiltration permeate. 
La rétention des protéines totales du lactosérum a été très élevée dès le début de la microfiltration (tableau I). Quelles que soient les conditions de vitesse tangentielle (V) et de pression transmembranaire (Pt) la rétention initiale était $>0,55$ et augmentait dans certains cas à $>0,80$ à un FCV de $3 X$ (fig 7). Les facteurs affectant significativement $(P<0,05)$ la rétention des protéines totales sont: $\mathrm{FCV}$ et $\mathrm{Pt}$, $\mathrm{FCV}^{2}$ et $\mathrm{Pt}^{2}, \mathrm{Pt}^{3}$ et les interactions $\mathrm{VX}$ $\mathrm{Pt}^{2}, \mathrm{~V}^{2} \times \mathrm{Pt}^{2}$ et $\mathrm{V}^{2} \times \mathrm{FCV}$.

La $\beta$-lactoglobuline du lactosérum est retenue par la membrane $0,22 \mu \mathrm{m}$ dès le début de la microfiltration ; la rétention varie entre 0,54 et 0,71 selon les conditions hydrodynamiques pour s'élever à 0,72 à 0,86 à FCV 3 (fig 8). Les facteurs affectant significativement $(P<0,05)$ la rétention de la $\beta$ lactoglobuline sont: $\mathrm{FCV}$ et $\mathrm{Pt}, \mathrm{FCV}^{2}$ et $\mathrm{Pt}^{2}$ et $\mathrm{Pt}^{3}$. Aucune interaction n'est significative.

L' $\alpha$-lactalbumine est la protéine la moins retenue par la membrane avec une rétention minimale de 0,39 s'élevant à un maximum de 0,68 (fig 9). Les facteurs significatifs $(P<0,05)$ dans l'évolution de la rétention de l' $\alpha$-lactalbumine sont les effets simples du FCV et de Pt.

Tableau I. Composition (\%) en protéines totales, $\beta$-lactoglobuline et $\alpha$-lactalbumine des rétentats et perméats lors de la microfiltration de lactosérum sur membranes d'alumine.

Total proteins, $\beta$-lactoglobulin and $\alpha$-lactalbumin content (\%) of the retentates and permeates during microfiltration of whey on alumina membranes.

\begin{tabular}{|c|c|c|c|c|c|c|c|c|c|}
\hline & \multicolumn{3}{|c|}{ Protéines totales $†$} & \multicolumn{3}{|c|}{$\beta$-lactoglobuline $t+$} & \multicolumn{3}{|c|}{$\alpha$-lactalbumine $t+$} \\
\hline & FCV 1 & FCV 2 & FCV 3 & FCV 1 & FCV 2 & FCV 3 & FCV 1 & FCV 2 & FCV 3 \\
\hline \multicolumn{10}{|c|}{$V=2 \mathrm{~m} \mathrm{~s}^{-1}$} \\
\hline $\begin{array}{l}\text { Rétentat } \\
\text { Pp1 } \\
\text { Pp2 } \\
\text { Pp3 } \\
\text { Pp4 }\end{array}$ & $\begin{array}{l}1,06 \\
0,38 \\
0,40 \\
0,45 \\
0,45\end{array}$ & $\begin{array}{l}1,52 \\
0,39 \\
0,42 \\
0,49 \\
0,52\end{array}$ & $\begin{array}{l}2,00 \\
0,42 \\
0,45 \\
0,53 \\
0,56\end{array}$ & $\begin{array}{l}0,59 \\
0,19 \\
0,21 \\
0,24 \\
0,24\end{array}$ & $\begin{array}{l}0,87 \\
0,19 \\
0,21 \\
0,26 \\
0,27\end{array}$ & $\begin{array}{l}1,17 \\
0,21 \\
0,23 \\
0,29 \\
0,30\end{array}$ & $\begin{array}{l}0,14 \\
0,07 \\
0,08 \\
0,08 \\
0,08\end{array}$ & $\begin{array}{l}0,17 \\
0,09 \\
0,09 \\
0,09 \\
0,09\end{array}$ & $\begin{array}{l}0,22 \\
0,09 \\
0,10 \\
0,09 \\
0,09\end{array}$ \\
\hline \multicolumn{10}{|c|}{$\mathrm{V}=3 \mathrm{~m} \mathrm{~s}^{-1}$} \\
\hline $\begin{array}{c}\text { Rétentat } \\
\text { Pp1 } \\
\text { Pp2 } \\
\text { Pp3 } \\
\text { Pp4 }\end{array}$ & $\begin{array}{l}1,05 \\
0,39 \\
0,40 \\
0,45 \\
0,46\end{array}$ & $\begin{array}{l}1,55 \\
0,39 \\
0,40 \\
0,48 \\
0,50\end{array}$ & $\begin{array}{l}2,11 \\
0,42 \\
0,43 \\
0,52 \\
0,55\end{array}$ & $\begin{array}{l}0,60 \\
0,20 \\
0,21 \\
0,24 \\
0,25\end{array}$ & $\begin{array}{l}0,88 \\
0,19 \\
0,20 \\
0,26 \\
0,27\end{array}$ & $\begin{array}{l}1,19 \\
0,21 \\
0,22 \\
0,28 \\
0,29\end{array}$ & $\begin{array}{l}0,14 \\
0,08 \\
0,08 \\
0,08 \\
0,08\end{array}$ & $\begin{array}{l}0,19 \\
0,08 \\
0,09 \\
0,09 \\
0,09\end{array}$ & $\begin{array}{l}0,24 \\
0,09 \\
0,09 \\
0,09 \\
0,09\end{array}$ \\
\hline \multicolumn{10}{|c|}{$\mathrm{V}=4 \mathrm{~m} \mathrm{~s}^{-1}$} \\
\hline $\begin{array}{l}\text { Rétentat } \\
\text { Pp1 } \\
\text { Pp2 } \\
\text { Pp3 } \\
\text { Pp4 }\end{array}$ & $\begin{array}{l}1,07 \\
0,39 \\
0,40 \\
0,44 \\
0,46\end{array}$ & $\begin{array}{l}1,57 \\
0,39 \\
0,40 \\
0,47 \\
0,50\end{array}$ & $\begin{array}{l}2,11 \\
0,42 \\
0,43 \\
0,51 \\
0,55\end{array}$ & $\begin{array}{l}0,59 \\
0,19 \\
0,20 \\
0,23 \\
0,24\end{array}$ & $\begin{array}{l}0,89 \\
0,17 \\
0,19 \\
0,23 \\
0,26\end{array}$ & $\begin{array}{l}1,17 \\
0,18 \\
0,20 \\
0,26 \\
0,28\end{array}$ & $\begin{array}{l}0,15 \\
0,08 \\
0,08 \\
0,08 \\
0,08\end{array}$ & $\begin{array}{l}0,20 \\
0,10 \\
0,10 \\
0,09 \\
0,10\end{array}$ & $\begin{array}{l}0,27 \\
0,10 \\
0,10 \\
0,10 \\
0,10\end{array}$ \\
\hline
\end{tabular}

† : moyennes de trois répétitions; †† : moyennes de deux répétitions ; Pp1 : perméat à Pt de $179 \mathrm{kPa} ; \mathrm{Pp} 2$ : perméat à Pt de $138 \mathrm{kPa}$; Pp3 : perméat à Pt de $97 \mathrm{kPa}$; Pp4 : perméat à Pt de $55 \mathrm{kPa}$; V : vitesse tangentielle. 


\section{DISCUSSION}

Les résultats obtenus par l'étude des résistances hydrauliques montrent une dépendance de Rm des membranes en fonction de la pression. L'équation de Poiseuille a été développée pour exprimer le flux dans un capillaire droit, lisse avec un écoulement en régime laminaire d'un fluide visqueux et incompressible. De même, la loi de Darcy décrit bien le flux de perméation à travers un milieu filtrant sous un écoulement en régime laminaire. En pratique, la structure des membranes de microfiltration en alumine (Charpin et al, 1991) suggère qu'il y a présence de pores tortueux et non lisses dans lesquels le régime d'écoulement est difficile à prédire. Dans ces conditions, les valeurs élevées de flux de perméation à l'eau (de l'ordre du millier de $1 \mathrm{~h}^{-1} \mathrm{~m}^{-2}$ ) des membranes propres laissent penser que le

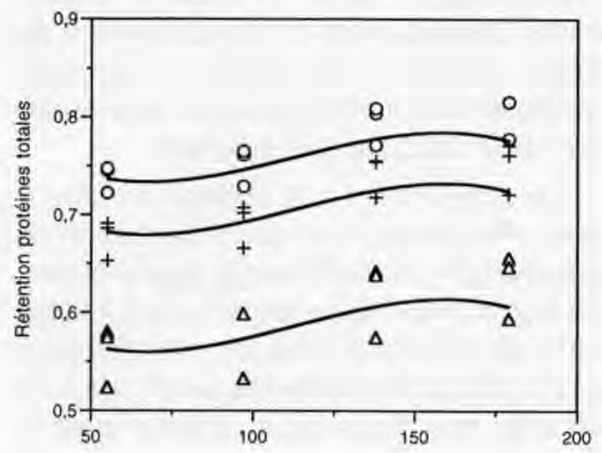

Pression transmembranaire (KPa)

Fig 7. Évolution de la rétention des protéines totales avec la pression transmembranaire et le facteur de concentration $(\Delta: 1 \mathrm{X},+: 2 \mathrm{X}, \mathrm{O}: 3 \mathrm{X})$ lors de la microfiltration de lactosérum à une vitesse tangentielle de $3 \mathrm{~m} \mathrm{~s}^{-1}$ sur membrane minérale. Courbe continue : modèle de régression avec $R^{2}$ $=0,927$. Points: résultats expérimentaux.

Evolution of the total protein retention with transmembrane pressure and concentration factor ( $\Delta$ : $1 X,+: 2 X, 0: 3 X)$ during microfiltration of whey at a tangential flow of $3 \mathrm{~m} \mathrm{~s}^{-1}$ on mineral membranes. Continuous curve: regression model with $R^{2}=0.927$. Points; experimental results. régime d'écoulement dans les pores n'est peut-être pas laminaire. Alors la relation entre le flux et la pression qui est linéaire tant que l'écoulement dans les pores est en régime laminaire change pour une relation où le flux varie avec la racine carrée de la pression en régime complètement turbulent (Munson et al, 1990). Nous supposons ainsi que lors de la filtration d'eau sur membranes de microfiltration propres (à flux de perméation élevé) l'écoulement pourrait ne pas être en régime laminaire dans les pores du microfiltre tel qu'identifié par la dépendance de la résistance membranaire face à la pression transmembranaire.

La nature chargée du matériau membranaire le rend susceptible de se comporter en échangeur d'ions et plus le flux à l'eau sera élevé, plus les échanges d'ions seront possibles. Le résultat est que les 4 membranes n'ont peut-être plus le même état de surface

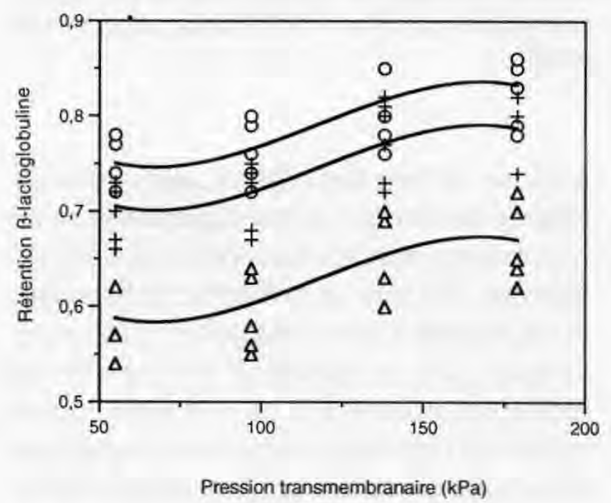

Fig 8. Évolution de la rétention de la $\beta$-lactoglobuline avec la pression transmembranaire et le facteur de concentration $(\Delta: 1 \mathrm{X},+: 2 \mathrm{X}, \mathrm{O}: 3 \mathrm{X})$ lors de la microfiltration de lactosérum sur membrane minérale. Courbe continue : modèle de régression avec $R^{2}=0,833$. Points : résultats expérimentaux.

Evolution of the $\beta$-lactoglobulin retention with transmembrane pressure and concentration factor $(\Delta: 1 X,+: 2 X, 0: 3 X)$ during microfiltration of whey on mineral membranes. Continuous curve: regression model with $R^{2}=0.833$. Points: experimental results. 


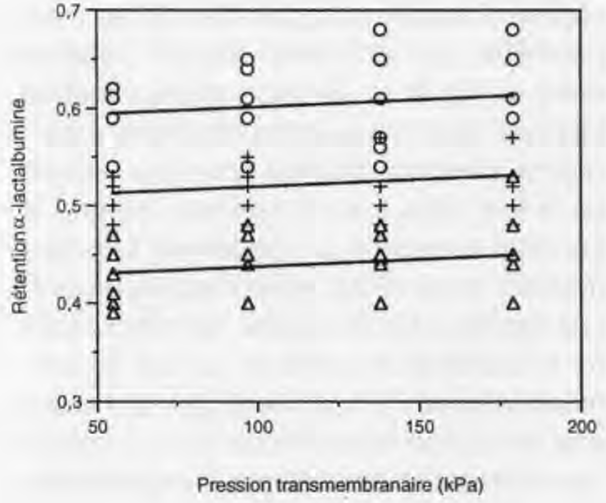

Fig 9. Evolution de la rétention de l' $\alpha$-lactalbumine avec la pression transmembranaire et le facteur de concentration $(\Delta: 1 \mathrm{X},+: 2 \mathrm{X}, 0: 3 \mathrm{X}$ ) lors de la microfiltration de lactosérum sur membrane minérale. Courbe continue : modèle de régression avec $R^{2}=0,798$. Points : résultats expérimentaux. Evolution of the $\alpha$-lactalbumin retention with transmembrane pressure and concentration factor ( $\Delta$ : $1 X,+: 2 X, 0: 3 X)$ during microfiltration of whey on mineral membranes. Continuous curve: regression model with $R^{2}=0.798$. Points: experimental results.

à cause de leur flux différent d'où cette différence de résistance membranaire entre les membranes opérant sous des pressions différentes. De plus, la présence d'effets électrocinétiques (Opong et Zydney, 1991) provoqués par le passage d'ions (ou de molécules polaires) dans la double couche diffuse de l'interface membrane-solvant peut provoquer un contre-courant électro-osmotique qui augmente la résistance hydraulique.

II est généralement admis (Merin et Daufin, 1990) que la microfiltration doit être réalisée à haute vitesse tangentielle pour limiter la concentration de polarisation et le gâteau de filtration, en surface du filtre. Dans cette optique, les conditions examinées montrent que la concentration de polarisation n'est pas le facteur le plus important dans le contrôle du flux de perméation puisqu'une augmentation du simple au double de la vitesse tangentielle (ie que le nombre de Reynolds double) ne provoque qu'une augmentation d'environ 1,25 fois du flux de perméation.

Lahoussine-Turcaud et al (1990) suggèrent que selon la taille des particules colmatantes, le flux diffusif et le cisaillement à la surface du filtre décolmateraient le filtre pendant que le flux convectif, généré par la pression transmembranaire, augmenterait le colmatage. Ainsi l'augmentation du flux convectif vers la surface de la membrane apporte plus de solutés que le cisaillement ne peut déloger qui dès le début de la filtration augmentent le colmatage.

Le passage d'un fluide dans un pore expose les protéines à des forces de cisaillement qui déforment ou réarrangent leur conformation. Ces protéines seraient alors plus susceptibles de s'adsorber et de former des agrégats affectant la perméabilité et la porosité. De plus, Opong et Zydney (1991) suggèrent que les couches de protéines déposées ont un comportement de milieu compressible qui lors d'une augmentation de Pt entraînera une augmentation de la résistance hydraulique.

Le cisaillement à la surface du filtre a pour effet de diminuer la concentration de polarisation d'où cette légère augmentation de flux avec la vitesse tangentielle. La faible différence entre les flux de microfiltration aux 4 pressions examinées serait due à un blocage des pores qui augmente avec la pression.

Meireles et al (1991) suggèrent qu'en ultrafiltration lors de filtration de $\mathrm{SAB}$, des combinaisons de paramètres tels : température, vitesse tangentielle, concentration et temps, pourraient conduire à la dénaturation (ie agrégation et polymérisation) des protéines. Les fines particules produites pourraient éventuellement pénétrer la couche filtrante d'une membrane de microfiltration et en bloquer les pores. De même, Bowen et Gan (1992) suggèrent que les phénomènes de cisaillement à la surface et dans les pores du filtre provoqueraient une dépo- 
sition sur les parois des pores de protéines déformées par le cisaillement. Cela se reflète dans le Rh du système. Celui-ci augmente au même rythme que la pression transmembranaire; de plus, l'augmentation de la vitesse tangentielle ne provoque qu'une faible diminution de $\mathrm{Rh}$. Ce qui traduit la présence d'un colmatage interne important par rapport au colmatage en surface.

L'équation de modélisation de $\mathrm{Rh}$ indique la dépendance de $\mathrm{Rh}$ face à la vitesse tangentielle, au facteur de concentration et à la pression transmembranaire qui est le facteur le plus important. On peut supposer qu'une vitesse tangentielle élevée créera plus de turbulence en surface du filtre permettant une limitation de la concentration de polarisation et du gâteau de filtration, d'où une diminution de Rh. L'augmentation de la concentration des solutés favorise les interactions solutéssolutés et solutés-membrane augmentant ainsi la possibilité d'adsorption multicouche et la possibilité de blocage des pores d'où une augmentation de Rh avec FCV. Une pression transmembranaire élevée augmentera les chances de blocage des pores en poussant et en entraînant plus profondément dans la matrice filtrante les particules de solutés d'où là aussi une augmentation de Rh.

L'analyse statistique de Rc a fait apparaître des interactions significatives entre la vitesse tangentielle (V) et la pression transmembranaire $(\mathrm{Pt})$ contrairement à $\mathrm{Rh}$ où il n'y avait que des effets simples de ces variables. Malgré ces interactions, $\mathrm{Pt}$ a beaucoup plus d'effet sur le colmatage que $V$ (fig 5). Cela confirme la présence d'un colmatage interne important surtout dû au blocage des pores. Puisque l'adsorption de soluté est une réaction physico-chimique qui dépend peu des conditions hydrodynamiques et que la turbulence de l'écoulement causée par $V$ ne fait varier que légèrement Rc, d'autres facteurs comme le blocage des pores peuvent alors augmenter Rc. L'augmentation du flux convectif causé par une pression plus élevée entraîne une plus grande quantité de solutés plus profondément dans la matrice microporeuse.

L'examen des surfaces décrivant Log Rc suggère que l'utilisation de faibles pressions transmembranaires avec des vitesses de balayage élevées, telles qu'utilisées en pratique industrielle, minimise la résistance hydraulique causée par le colmatage.

Nos résultats de rétention protéique montrent une rétention totale de la $\mathrm{SAB}$ et partielle de la $\beta$-lactoglobuline et de l' $\alpha$-lactalbumine, la $\beta$-lactoglobuline étant légèrement plus retenue que l' $\alpha$-lactalbumine. Ces résultats montrent les mêmes tendances que ceux rapportés par Attia et al (1991a et b), Pierre et al (1992) et Gésan (1993) pour la $\beta$-lactoglobuline et l' $\alpha$-lactalbumine, bien que dans ces derniers cas, les rétentions soient plus faibles. Certains de ces résultats ont cependant été obtenus avec des fluides différents et sur des systèmes légèrement différents, produisant des pressions transmembranaires beaucoup plus faibles, ce qui permet des rétentions également plus faibles.

Malgré ces différences, le fait est que pour ces protéines les membranes de $0,22 \mu \mathrm{m}$, bien qu'ayant un diamètre moyen de pore 20 fois supérieur à la plus grande dimension de la protéine la plus volumineuse montrent une forte rétention même pour les petites protéines.

Nos analyses statistiques indiquent que la concentration et surtout la pression ont des effets sur la rétention protéique. Opong et Zydney (1991) rapportent que la pression hydrostatique et la contrainte de cisaillement exercées par le passage du fluide induisent une déformation ou réarrangement des protéines provoquant la formation d'un dépôt sur les parois des pores, entraînant ainsi une diminution de la perméabilité et de la porosité. L'observation que la vitesse de balayage n'a aucune influence directe sur la rétention appuie l'idée d'un colmatage interne de la matrice microporeuse qui contrôlerait la rétention des protéines. 
Selon Bowen et Gan (1991a), dans le cas de la microfiltration de SAB, l'adsorption d'une monocouche de protéines ne peut pas à elle seule provoquer un rétrécissement du diamètre des pores suffisant pour induire une chute de perméabilité importante. D'autres phénomènes doivent aussi participer à ce processus. Ces mêmes auteurs ont ainsi conclu qu'il se produit de l'adsorption et déposition en multicouche en profondeur du filtre. Lors du passage des protéines, il pourrait y avoir collision entre les protéines solubles et la monocouche adsorbée foumissant assez d'énergie pour la formation de complexes susceptibles de se déposer sur les parois du pore. Par ailleurs, selon Kim et al (1993), le cisaillement induit dans le fluide, à concentration élevée de solutés (ie près de la membrane), pourrait déformer ou déplier les protéines, ce qui augmenterait les possibilités de flocculation dues aux collisions entre particules. Ces phénomènes d'agrégation ou polymérisation (Meireles et al, 1991) ou de dénaturation (Bowen et Gan, 1992) seraient dus à la distorsion et à la déformation de la configuration normale des protéines sous l'effet de la contrainte de cisaillement à l'interface membrane-solution à la surface et dans les pores de la membrane.

Lee et Merson (1975) ont mis en évidence par microscopie électronique la présence de feuillets de $S A B$ et de $\beta$-lactoglobuline et de particules sphériques d' $\alpha$-lactalbumine lors de la microfiltration de solutions protéiques sur membrane Nucléopore de $0,4 \mu \mathrm{m}$. Ces modifications protéiques pourraient provenir de combinaisons de différentes conditions hydrodynamiques et physicochimiques (vitesse de balayage, pression, température, concentration, etc) (Meireles et al, 1991 ; Kim et al, 1993), ce qui rendrait les protéines plus susceptibles d'obstruer partiellement les pores provoquant directement un effet sur la perméabilité et la sélectivité des membranes (Bowen et Hughes, 1990).

Si ces phénomènes de dénaturation existent lors de la filtration de solutions de pro- téines pures, il est alors possible que cela se produise lors de la microfiltration de lactosérum. II existerait donc une combinaison de différents facteurs hydrodynamiques et physico-chimiques conduisant à une dénaturation ou agrégation. Le phénomène provoquerait un colmatage élevé et donc une rétention supérieure se reflétant dans les interactions significatives que l'on retrouve entre V, Pt et FCV pour la rétention des protéines totales.

\section{CONCLUSIONS}

Lors de la microfiltration tangentielle de lactosérum, la membrane d'alumine se comporte comme un filtre en profondeur où la principale cause de colmatage est le blocage des pores par les solutés très probablement sous forme associée entre eux et avec le matériel membranaire. La rétention des protéines résulterait aussi du colmatage interne de la membrane, tel que le suggère la non dépendance directe de la rétention par rapport à la vitesse tangentielle. Les interactions entre les facteurs vitesse tangentielle $\mathrm{x}$ facteur de concentration volumique $x$ pression transmembranaire amènent à penser que la rétention dépend des conditions hydrodynamiques en plus des conditions physico-chimiques des fluides. La combinaison de ces facteurs pourrait provoquer la dénaturation ou la réorganisation des protéines (agrégation ou polymérisation) alors susceptibles de s'adsorber et de se déposer à l'intérieur des pores provoquant une diminution de la perméabilité et de la porosité membranaire.

\section{RÉFÉRENCES}

Attia H. Bennasar M, Tarodo De La Fuente B (1991a) Study of the fouling of inorganic membranes by acidified milks using scanning electron microscopy and electrophoresis. I. Membrane with pore diameter 0.2 $\mu \mathrm{m}$. J Dairy Res 58, 39-50 
Attia H, Bennasar M, Tarodo De La Fuente B (1991b) Study of the fouling of inorganic membranes by acidified milks using scanning electron microscopy and electrophoresis. II. Membrane with pore diameter $0.8 \mu \mathrm{m}$. J Dairy Res $58,51-65$

Azbel DS, Cheremisinoif NP (1983) Fluid mechanics and unit operations. Ann Arbor Science Publ, USA

Belleville MP. Brillouet JM, Tarodo De La Fuente B, Moutounet M (1992) Fouling colloids during microporous alumina membrane filtration of wine. $J$ Food Sci $57,396-400$

Bowen WR, Hughes DT (1990) Properties of microfiltration membranes. Part 2. Adsorption of bovine serum albumin at aluminium oxide membranes. J Membrane Sci 51, 189-200

Bowen WR, Gan Q (1991a) Properties of microfiltration membranes: Flux loss during constant pressure permeation of bovine serum albumin. Biotechnol Bioeng $38,688-696$

Bowen WR, Gan Q (1991b) Properties of microfiltration membranes: Adsorption of bovine serum albumin at polyvinylidene fluoride membranes. I Colloid Interface Sci 144, 254-262

Bowen WR, Gan Q (1992) Properties of microfiltration membranes: The effects of adsorption and shear on the recovery of an enzyme. Biotechnol Bioeng 40 , 491-497

Bowen WR, Gan Q (1993) Microfiltration of protein solutions at thin film composite membranes. J Membrane Sci $80,165-173$

Brew K, Grobler JA (1992) $\alpha$-lactalbumin. In: Advanced dairy chemistry-1: Proteins (Fox PF, ed). Elsevier, London

Chan KK, Brownstein AM (1991) Ceramic membranesGrowth prospects and opportunities. Ceram Bull 70 , 703-707

Charpin J, Burggraaf AJ, Cot L (1991) A survey of ceramic membranes for separations in liquid and gaseous media. Ind Ceram 11, 84-89

Cheryan M (1986) Ultrafiltration handbook. Technomic Publishing $\mathrm{Co}$, Lancaster $\mathrm{Pa}$, USA

Daufin G, Merin U, Kerhervé FL, Labbé JP, Quémerais A, Bousser C (1992) Efficiency of cleaning agents for an inorganic membrane after milk ultrafiltration. $J$ Dairy Res 59, 29-38

Gésan G (1993) Microfiltration tangentielle de lactosérum doux prétraité. Mécanismes, conduite opératoire. Thèse de Doctorat, université Rennes I

Hambling SG, McAlpine AS, Sawyer L (1992) B-lactoglobulin. In: Advanced dairy chemistry-1: Proteins (Fox PF, ed). Elsevier, London

Kelly ST, Opong WS, Zydney AL (1993) The influence of protein aggregates on the fouling of microfiltration membranes during stirred cell filtration. J Membrane Sci $80,175-187$

Kim KJ, Chen V, Fane AG (1993) Some factors determining protein aggregation during ultrafiltration. Biotechnol Bioeng 42, 260-265
Lahoussine-Turcaud V, Wiesner MR, Bottero JY (1990) Fouling in tangential-flow ultrafiltration: The effect of colloid size and coagulation pretreatment. $J$ Membrane Sci 52, 173-190

Lee DN, Merson RL (1975) Examination of cottage cheese whey proteins by scanning electron microscopy: Relationship to membrane fouling during ultrafiltration. J Dairy Sci 58, 1423-1432

Marshall KR, Harper WS (1988) Whey protein concentrates. Int Dairy Fed Bull 233, 21-32

Meireles M, Aimar P, Sanchez V (1991) Albumin denaturation during ultrafiltration: Effects of operating conditions and consequences on membrane fouling. Biotechnol Bioeng 38, 528-534

Merin U, Daufin G (1990) Crossflow microfiltration in the dairy industry: State-of-the-art. Lait 70, 281-291

Mietton-Peuchot M, Milisic V, Ben Aim R (1984) Microfiltration tangentielle des boissons. Influence des caractéristiques hydrodynamiques. Lait 64, 121-128

Munson BR, Young DF, Okiishi TH (1990) Fundamentals of fluid mechanics. John Wiley \& sons, New-York

Nilsson JL (1990) Protein fouling of UF membranes: Causes and consequences. J Membrane Sci 52 , $121-142$

Olesen N, Jensen F (1989) Microfiltration. The influence of operation parameters on the process. Milchwissenschaft $44,476-479$

Opong WS, Zydney AL (1991) Hydraulic permeability of protein layers deposited during ultrafiltration. $J \mathrm{Col}$ loid Interface Sci 142, 41-60

Pierre A, Fauquant J, Le Graet Y, Piot M, Maubois JL (1992) Préparation de phosphocaséinate natif par microfiltration sur membrane Lait $72,461-474$

Piot M, Maubois JL, Schaegis P, Veyre R, Luccioni A (1984) Microfiltration en flux tangentiel des lactosérums de fromagerie. Lait $64,102-120$

Steel RGD, Torrie JH (1980) Principles and procedures of statistics. McGraw-Hill, New-York

Taddei C, Aimar P, Daufin G, Sanchez V (1988) Factors affecting fouling of an inorganic membrane during sweet whey ultrafiltration. Lait $68,157-176$

Taddei C, Daufin G, Aimar P, Sanchez V (1989) Role of some whey components on mass transfer in ultrafiltration. Biotechnol Bioeng 34, 171-179

Trouvé E, Maubois JL, Piot M et al (1991) Rétention de différentes espèces microbiennes lors de l'épuration du lait par microfiltration en flux tangentiel. Lait $71,1-13$

Van Der Horst H C, Hanemaaijer J H (1990) Cross-flow microfiltration in the food industry. State of the art. Desalination 77, 235-258

Vétier C, Bennasar M, Tarodo De La Fuente B (1986) Etude des interactions entre constituants du lait et membranes minérales de microfiltration. Lait 66 269-287

Warren RK, Macdonald DG, Hill GA (1992) Cross-flow microfiltration of Saccharomyces cerevisiae. Process Biochem 26, 337-342 